\title{
Duration of Inhibition of Ventral Tegmental Area Dopamine Neurons Encodes a Level of Conditioned Fear
}

\author{
Boris Mileykovskiy and Marisela Morales \\ National Institute on Drug Abuse, Intramural Research Program, Neuronal Networks Section, Baltimore, Maryland 21224
}

It is widely accepted that midbrain dopamine (DA) neurons encode actual and expected reward values by phasic alterations in firing rate. However, how DA neurons encode negative events in the environment is still unclear because some DA neurons appear to be depressed and others excited by aversive stimuli. Here, we show that exposing fear-conditioned rats to stimuli predicting electrical shock elicited three types of biphasic responses, each of which contained an inhibitory pause, in neurochemically identified ventral tegmental area (VTA) DA neurons. The duration of the inhibitory pause in these responses of VTA DA neurons was in direct proportion to the increase in respiratory rate reflecting the level of conditioned fear. Our results suggest that the duration of inhibition of VTA DA neurons encodes negative emotional values of signals predicting aversive events in the environment.

\section{Introduction}

Dopamine (DA) neurons located in the ventral tegmental area (VTA) project to the amygdala, prefrontal cortex, nucleus accumbens, and hippocampus (Swanson, 1982; Gasbarri et al., 1994), structures known to be involved in the regulation of aversive behavior. Electrophysiological studies in both anesthetized and conscious animals showed that aversive stimuli either phasically excite or inhibit DA neurons (Mirenowicz and Schultz, 1996; Guarraci and Kapp, 1999; Ungless et al., 2004; Brischoux et al., 2009; Matsumoto and Hikosaka, 2009a), and that the type of response of DA neurons depends, to a certain degree, on their location within the midbrain. In particular, experiments in anesthetized rats have demonstrated that nociceptive electrical stimulation elicits inhibitory responses in neurochemically identified DA neurons located predominantly in the dorsal part of the VTA (Ungless et al., 2004), whereas ventral VTA DA neurons are excited by noxious stimulation (Brischoux et al., 2009). In conscious monkeys, excitatory response to an aversive air-puff and associated conditioned stimuli (CSs) were observed in putative DA neurons located in the substantia nigra pars compacta. This group of putative DA neurons also discharged in response to presentations of reward-predicting stimuli (Matsumoto and Hikosaka, 2009a). In contrast, putative VTA DA neurons, which were excited by rewarding stimuli, showed inhibitory responses to aversive stimuli, suggesting that these neurons participate in

\footnotetext{
Received Oct. 31, 2010; revised March 15, 2011; accepted March 17, 2011.

Author contributions: B.Y.M. designed research; B.Y.M. performed research; B.Y.M. and M.M. analyzed data; B.Y.M. and M.M. wrote the paper.

This research was supported by the Intramural Research Program of the National Institute on Drug Abuse. We thank Dr. Roy Wise for comments to this manuscript.

The authors declare no competing financial interests.

This article is freely available online through the J Neurosci Open Choice option.

Correspondence should be addressed to either Boris Y. Mileykovskiy or Marisela Morales, National Institute on

Drug Abuse, Intramural Research Program, Neuronal Network Section, 251 Bayview Boulevard, Baltimore, MD 21224. E-mail: mileykob@nida.nih.gov or mmorales@intra.nida.nih.gov.

DOI:10.1523/JNEUROSCI.5731-10.2011

Copyright $\odot 2011$ the authors $\quad 0270-6474 / 11 / 317471-06 \$ 15.00 / 0$
}

the encoding of both positive and negative emotional values (Schultz, 2007; Matsumoto and Hikosaka, 2009a).

It is still unclear how VTA DA neurons encode a broad range of aversive stimuli. Since these DA neurons have a low spontaneous firing rate and cease their activity in response to mild negative events (Mirenowicz and Schultz, 1996), there is the possibility that alterations in the duration of inhibition of DA neurons may encode responses to aversive stimuli, as was shown for negative reward prediction errors in monkeys (Bayer et al., 2007). However, previous studies have demonstrated that neurons electrophysiologically identified as VTA DA neurons showed bidirectional responses to aversive stimuli (Mirenowicz and Schultz, 1996; Guarraci and Kapp, 1999, Matsumoto and Hikosaka, 2009a). These reported excitatory and inhibitory responses of VTA DA neurons might be related to their distribution within the midbrain (Ungless et al., 2004; Brischoux et al., 2009) or their electrophysiological misidentification based solely on spike electrophysiological parameters. Juxtacellular Neurobiotin $(\mathrm{Nb})$ labeling of VTA neurons recorded in anesthetized rats has revealed that a large population of cells that does not express tyrosine hydroxylase (TH), a marker of DA neurons, has spike parameters similar to those of TH-positive neurons (Ungless et al., 2004; Luo et al., 2008; Brischoux et al., 2009). These data clearly demonstrate the need to combine electrophysiological and neurochemical criteria for the accurate identification of VTA DA neurons in conscious animals.

We have developed a novel methodological approach that allows juxtacellular labeling of neurons recorded in behaving rats and their subsequent neurochemical identification. By using this approach, we were able to record responses of neurochemically identified VTA DA neurons to aversive CSs paired with electrical shock in fearconditioned rats and to establish that the duration of inhibition of these DA neurons is modulated by fear in a monotonic manner.

\section{Materials and Methods}

Subjects. Experiments were performed in 23 male Sprague Dawley rats. Two plastic microdrive hubs, bipolar stimulating, and indifferent electrodes were fixed on the skull under general anesthesia (Equithesin, 3.3 
$\mathrm{ml} / \mathrm{kg}$, i.p.). Coordinates of the VTA and gigantocellular reticular nucleus were determined with the rat brain atlas of Paxinos and Watson (1997). Chronic EEG and neck EMG electrodes were additionally implanted in six control rats to assess the sleep-wakefulness states during habituation. All procedures and experiments were approved by the Animal Care and Use Committee of the National Institute on Drug Abuse and complied with the Public Health Service Policy on use of laboratory animals.

Training procedures. Before learning, rats were habituated to light restraint with a fabric hammock for 2-3 d (3-4 h daily). Our criteria for habituation were as follows: low frequency of defecation, lack of strong motor activity and freezing episodes, normal respiratory rate, and ability to fall asleep during restraint. Seventeen rats were fear conditioned using two different tones $(10 \mathrm{kHz}$ or $600 \mathrm{~Hz}, 70 \mathrm{~dB}, 3 \mathrm{~s})$. The first tone $\left(\mathrm{CS}^{+}\right)$, paired with subsequent electrical tail shock $(0.5-1.2 \mathrm{~mA}, 60 \mathrm{~Hz}, 1 \mathrm{~s})$, was delivered during a $30 \mathrm{~min}$ session with $50-60 \mathrm{~s}$ intertrial intervals. The second tone $\left(\mathrm{CS}^{-}\right)$, unpaired with shock, was presented 20-30 s after the $\mathrm{CS}^{+}$. Auditory frequencies for the $\mathrm{CS}^{+}$and $\mathrm{CS}^{-}$were counterbalanced across rats. For comparison, the same tones unpaired with electrical shock were presented to six naive rats habituated to light restraint. The respiratory rate was recorded to evaluate levels of fear evoked by the CSs or control tones (Frysztak and Neafsey, 1991; Ley, 1999; Gallego et al., 2001). For this purpose, a respiratory transducer (a miniature headphone without a cover) was placed between the body of a rat and the fabric hammock. EMG electrodes (50 $\mu \mathrm{m}$ stainless steel wires) were implanted bilaterally in the gastrocnemius muscles to record acoustic startle responses to the $\mathrm{CS}^{+}$and $\mathrm{CS}^{-}$. Training was stopped when rats accelerated breathing to 10 consecutive $\mathrm{CS}^{+}$presentations and did not respond to the $\mathrm{CS}^{-}$delivery. On the next day, the additional one or two conditioning sessions (10-15 $\mathrm{min}$ ) were performed before neuronal recording.

Recording and electrical stimulation. Recording of spikes and juxtacellular labeling of neurons were performed using Axoclamp 900A (Molecular Devices) and Model 1700 (A-M Systems) amplifiers. Micropipettes were filled with $4 \% \mathrm{Nb}$ dissolved in $0.5 \mathrm{M}$ potassium acetate. To obtain stable recording and juxtacellular labeling of neurons, we used micropipettes with thick tips $(2.0-3.0 \mu \mathrm{m}$ in diameter) and a sinusoidal shape of injection currents $(3-15 \mathrm{nA})$.

Electrical stimulation of the medial part of the gigantocellular reticular nucleus (Mileykovskiy et al., 2002) immobilized the rat for filling a cell with $\mathrm{Nb}$ after a recording session. As an application of standard rectangular stimulating pulses evoked strong electrical artifacts, which impeded the control of cell labeling, we used sinusoidal currents $(100-250 \mu \mathrm{A}, 25$ $\mathrm{Hz}, 5-15 \mathrm{~min}$ ) for electrical stimulation of the gigantocellular reticular nucleus. Agilent 33220A generator (Agilent Technologies) was chosen for stimulation because its output, isolated from the ground, substantially decreased electrical artifacts. Recording of spikes was performed in the frequency band from $100 \mathrm{~Hz}$ to $5 \mathrm{kHz}$. An additional filter $(300 \mathrm{~Hz}-5$ $\mathrm{kHz}$ ) was connected during juxtacellular labeling. The signal/noise ratio $>5 / 1$ allowed successful juxtacellular labeling of a single neuron. One or two neurons with similar discharge patterns were labeled on each side of the brain.

Immunohistochemical identification of neurons. VTA sections $(20 \mu \mathrm{m})$ were incubated in cyanine 2-conjugated streptavidin (1:200; Jackson ImmunoResearch) overnight. Nb-labeled neurons were visualized by epifluorescence using a Leica DMLB microscope. Sections containing $\mathrm{Nb}$-labeled neurons were incubated for $3 \mathrm{~h}$ in a mouse monoclonal anti-TH antibody (1:200; Millipore Bioscience Research Reagents), followed by $3 \mathrm{~h}$ incubation in a Texas Red dye-conjugated donkey antimouse antibody (1:50; Jackson ImmunoResearch).

Statistical analysis. The duration of inhibitory pauses of DA neurons, which showed inhibitory responses to the CSs, was calculated from the onset of the inhibitory pause to the appearance of the first spike (Bayer et al., 2007). In three DA neurons showing biphasic excitatory/inhibitory responses, the duration of inhibitory pauses was calculated from the last short latency spike to the appearance of the first spike. The firing rate following the $\mathrm{CS}^{+}$or $\mathrm{CS}^{-}$termination was determined for $1 \mathrm{~s}$ using peristimulus time histograms (100 ms bin) constructed for each DA neuron and compared with the baseline firing rate calculated for $3 \mathrm{~s}$ preceding the CS onset. The respiratory rate and firing rate of DA neu-
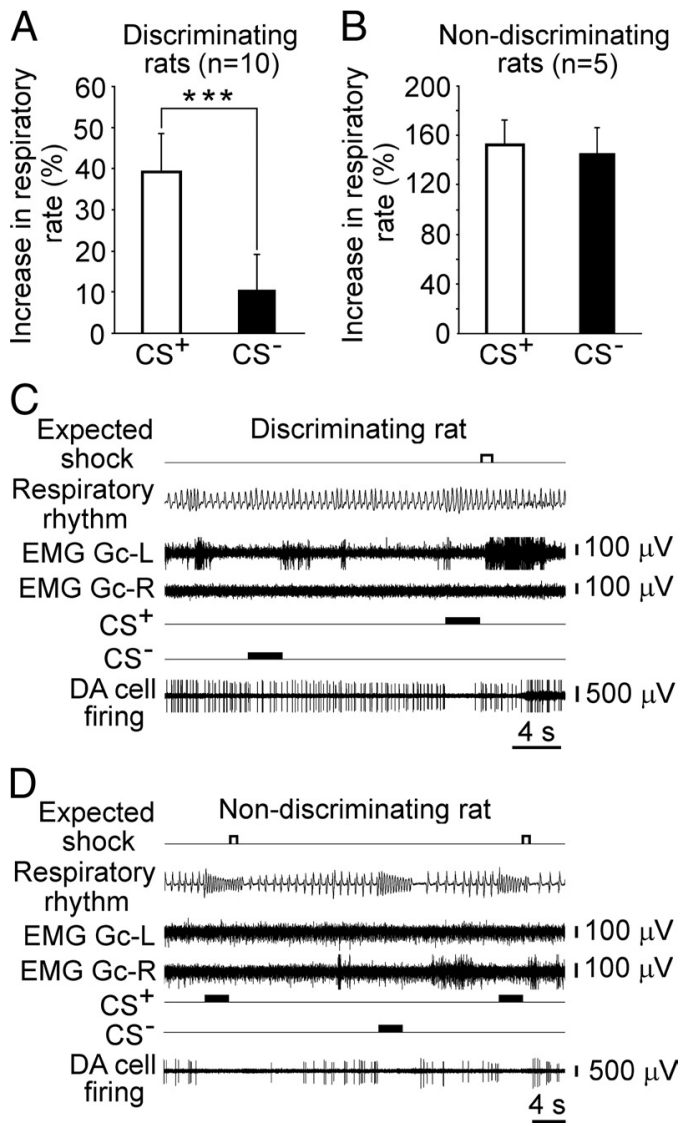

Figure 1. Alterations in respiratory rate and DA neuronal firing elicited by the $\mathrm{CS}^{+}$and $\mathrm{CS}^{-}$ $A$, Differential respiratory responses recorded during the initial six trials in rats $(n=12)$ discriminating between the $\mathrm{CS}^{+}$and $\mathrm{CS}{ }^{-}$. Paired $t$ test, $t=5.588,{ }^{* * *} p<0.0002, \mathrm{df}=11 . \boldsymbol{B}$, Similar increases in respiratory rate evoked by the $\mathrm{CS}^{+}$and $\mathrm{CS}^{-}$in non-discriminating rats $(n=5)$ during the initial six trials. Wilcoxon matched-pairs signed-rank test, $p=0.4375 . C, D$, Representative respiratory and DA neuronal inhibitory responses to the $C S^{+}$and $C S^{-}$recorded in discriminating $(\boldsymbol{C})$ and nondiscriminating $(\boldsymbol{D})$ rats. Electromyograms of the left (EMG GC-L) and right (EMG GC-R) gastrocnemius muscles. Auditory conditioned stimuli paired (CS ${ }^{+}$) and unpaired (CS ${ }^{-}$) with electrical tail shock.

rons were calculated for $3 \mathrm{~s}$ before (baseline) and after turning on $\mathrm{CS}^{+}, \mathrm{CS}^{-}$, or control auditory tones. The alterations in respiratory rate evoked by the CSs were indicated as a percentage of the baseline value. Freezing was defined as complete immobility of rats, excepting respiratory movements. Every second following the $\mathrm{CS}^{+}$or $\mathrm{CS}^{-}$onset, an experimenter evaluated the freezing or not freezing state of the rat. Two-tail probability value was used in all statistical tests. All data are presented as mean \pm SEM.

\section{Results}

After five to eight training sessions, 12 of the 17 fear-conditioned rats developed differential respiratory responses to the $\mathrm{CS}^{+}$and $\mathrm{CS}^{-}$, showing a higher increase in respiratory rate to the $\mathrm{CS}^{+}$ than to the $\mathrm{CS}^{-}$(Fig. $1 A, C$; supplemental Fig. S1 $A, B$, available at www.jneurosci.org as supplemental material). These discriminating rats expressed short freezing episodes after the $\mathrm{CS}^{+}$onset $(7.5 \pm 0.6 \mathrm{~s}$, minimum $=4.2 \mathrm{~s}$, maximum $=11.6 \mathrm{~s}, n=12)$ and a restored normal breathing rhythm $(1.81 \pm 0.04 \mathrm{cycle} / \mathrm{s}$, minimum $=1.54 \mathrm{cycle} / \mathrm{s}$, maximum $=2.2 \mathrm{cycle} / \mathrm{s}, n=12)$ before the subsequent $\mathrm{CS}^{-}$presentation. Five of the 17 rats did not discriminate between the $\mathrm{CS}^{+}$and $\mathrm{CS}^{-}$and exhibited strong breathing acceleration in response to both stimuli (Fig. $1 B, D$; supplemental Fig. $S 1 C, D$, available at www.jneurosci.org as supplemental material). During intertrial periods, these non-discriminating rats 

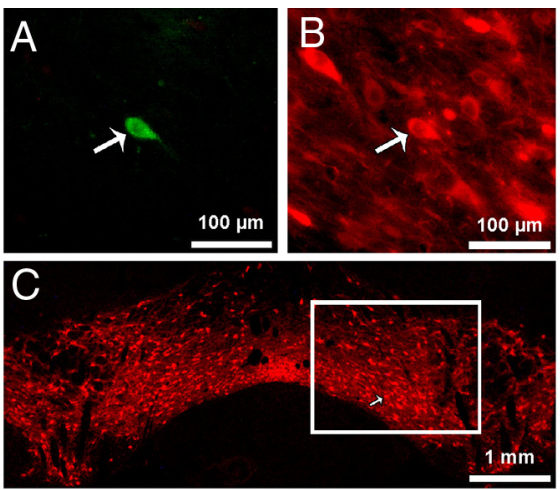

D

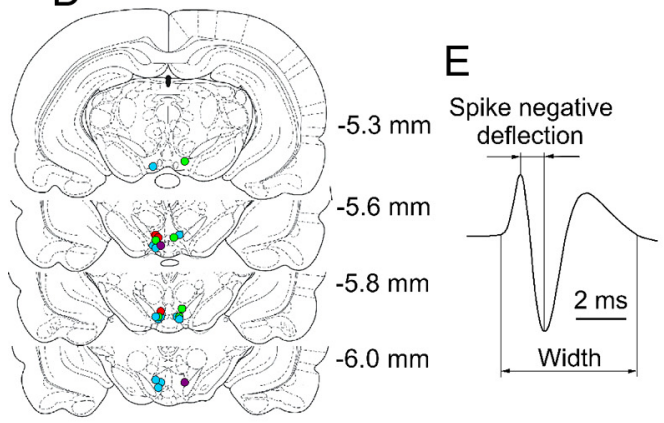

$\mathrm{F}$
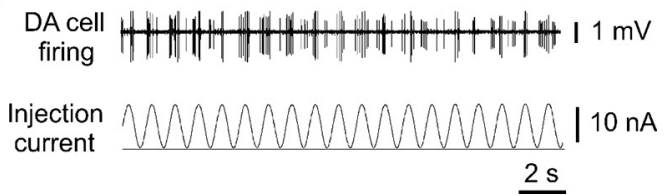

Figure 2. Neurochemical identification of DA neurons and their location in the VTA.A, AVTA neuron juxtacellularly labeled with $\mathrm{Nb}$. $\boldsymbol{B}$, This neuron expresses TH immunoreactivity. $\boldsymbol{C}$, The location of this TH-positive neuron in the ventral part of the VTA. D, Cumulative data showing the location of 23 recorded DA neurons in the VTA. Blue dots, DA neurons inhibited by the $\mathrm{CS}^{+}$; red dots, DA neurons showing biphasic excitatory/inhibitory responses; brown dots, DA neurons recorded in rats after fear extinction; green dots, DA neurons recorded in naive rats habituated to light restraint. $E$, A triphasic waveform of a DA neuronal spike. $F$, Modulation of the activity of a DA neuron during juxtacellular labeling with $\mathrm{Nb}$.

expressed continuous freezing behavior that was characterized by a slow respiratory rhythm $(1.17 \pm 0.03 \mathrm{cycle} / \mathrm{s}$, minimum $=0.85$ cycle/s, maximum $=1.48 \mathrm{cycle} / \mathrm{s}, n=5)$ alternating with short episodes of accelerated breathing.

Of 49 VTA neurons recorded during 9-13 trials and submitted to juxtacellular labeling, 32 cells $(65 \%)$ were successfully entrained by iontophoretic currents, filled with $\mathrm{Nb}$, and visualized in the brain sections. Twenty-three of the 32 VTA neurons expressed $\mathrm{TH}$ immunoreactivity, a selective marker of DA, and were predominantly located within the parabrachial pigmented and paranigral nuclei (Fig. $2 A-D$ ). These DA neurons were recorded in 23 rats: 15 fear-conditioned rats ( 10 discriminating and five non-discriminating), two discriminating rats after fear extinction, and six naive rats habituated to light restraint. Nine of the 32 VTA neurons were TH-negative (supplemental Fig. S2, available at www.jneurosci.org as supplemental material).

All TH-positive neurons had broad triphasic spikes (Fig. 2E) and slow irregular or bursting activity that clearly distinguished them from putative GABA neurons having narrow spikes and high firing rates. These discharge parameters, however, were insufficient to distinguish TH-positive neurons from $\mathrm{TH}$-negative neurons that had slower firing rates and broader spikes than pu-
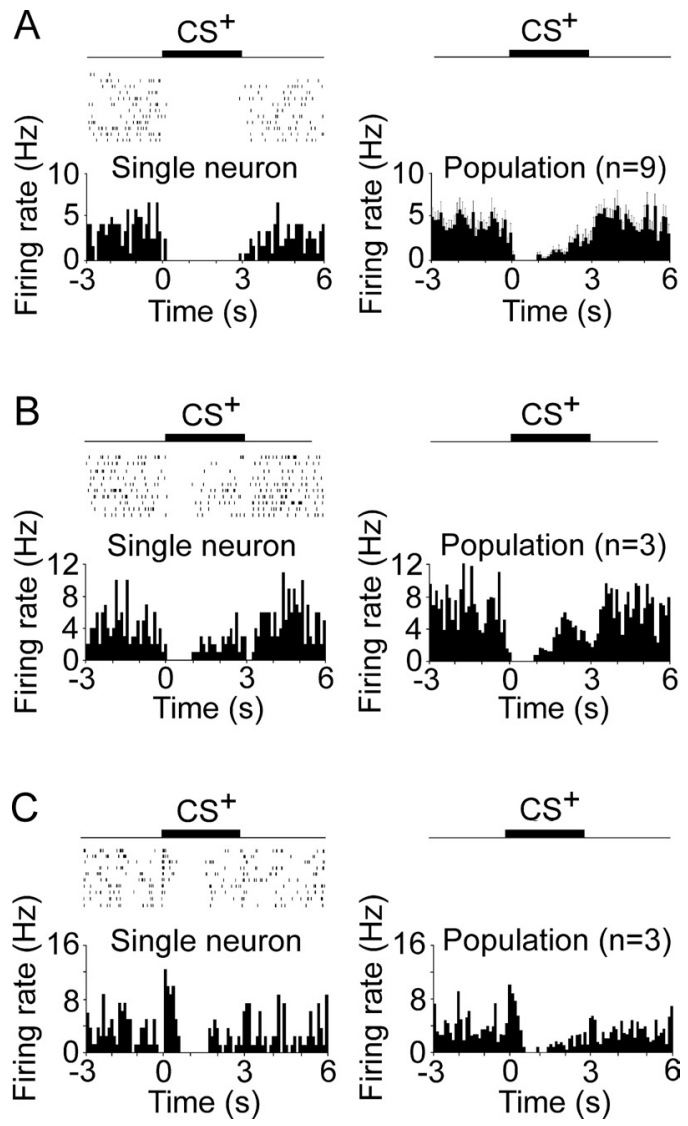

Figure 3. Three types of responses of VTA DA neurons to the $\mathrm{CS}^{+}$delivery. $\boldsymbol{A}$, Spike raster plot and peristimulus time histograms ( $100 \mathrm{~ms}$ bin) illustrating continuous inhibition of DA neurons to the onset of the $C S^{+}$. $\boldsymbol{B}$, Spike raster plot and peristimulus time histograms $(100 \mathrm{~ms}$ bin) illustrating biphasic inhibition of DA neurons to the $\mathrm{CS}^{+}$onset and offset. $C$, Spike raster plot and peristimulus time histograms (100 ms bin) illustrating biphasic excitatory/inhibitory responses of DA neurons to the $\mathrm{CS}^{+}$.

tative GABA neurons (supplemental Fig. $S 3 A-D$, available at www.jneurosci.org as supplemental material). Durations of spike negative deflections (Fig. 2E), however, were significantly different between these two cell groups (supplemental Fig. S3E, available at www.jneurosci.org as supplemental material).

Presentations of the $\mathrm{CS}^{+}$that evoked respiratory acceleration in fear-conditioned rats elicited three types of responses in VTA DA neurons $(n=15)$. In particular, nine of the 15 DA neurons ceased their discharges in response to the onset of the $\mathrm{CS}^{+}$(Fig. $3 A$ ). The average firing rate of these nine neurons during the $\mathrm{CS}^{+}$delivery significantly decreased from $4.1 \pm 0.5 \mathrm{~Hz}$ to $0.75 \pm 0.35 \mathrm{~Hz}$ (paired $t$ test, $t=9.87, p<0.00001$, df $=8$ ), with the subsequent increase by $42 \pm 14 \%$ (minimum $=4 \%$, maximum $=65 \%, n=9$; paired $t$ test, $t=2.58, p=0.01557, \mathrm{df}=8$ ) compared with baseline. In response to the $\mathrm{CS}^{-}$, the average firing rate of these DA neurons altered insignificantly $(4.1 \pm 0.5$ $\mathrm{Hz}$ vs $3.6 \pm 0.4 \mathrm{~Hz}$, paired $t$ test, $t=1.01, p=0.17061, \mathrm{df}=8)$. Three of the $15 \mathrm{DA}$ neurons decreased their activity to both the $\mathrm{CS}^{+}$onset and offset (Fig. $3 \mathrm{~B}$ ). The inhibitory responses in these $12 \mathrm{DA}$ neurons to the $\mathrm{CS}^{+}$onset had an average latency of $78.1 \pm$ $7.0 \mathrm{~ms}$ ( minimum $=43 \mathrm{~ms}$, maximum $=139 \mathrm{~ms}$ ) and an average duration of inhibitory pauses of $2.8 \pm 0.4 \mathrm{~s}$ (minimum $=0.9 \mathrm{~s}$, maximum $=5.9 \mathrm{~s}$ ). The other three of the 15 DA neurons showed biphasic excitatory/inhibitory responses consisting of two to five short-latency $(18-45 \mathrm{~ms})$ spikes followed by inhibitory pauses with durations from 0.5 to $3.7 \mathrm{~s}$ (Fig. $3 C$ ). The occurrence of 

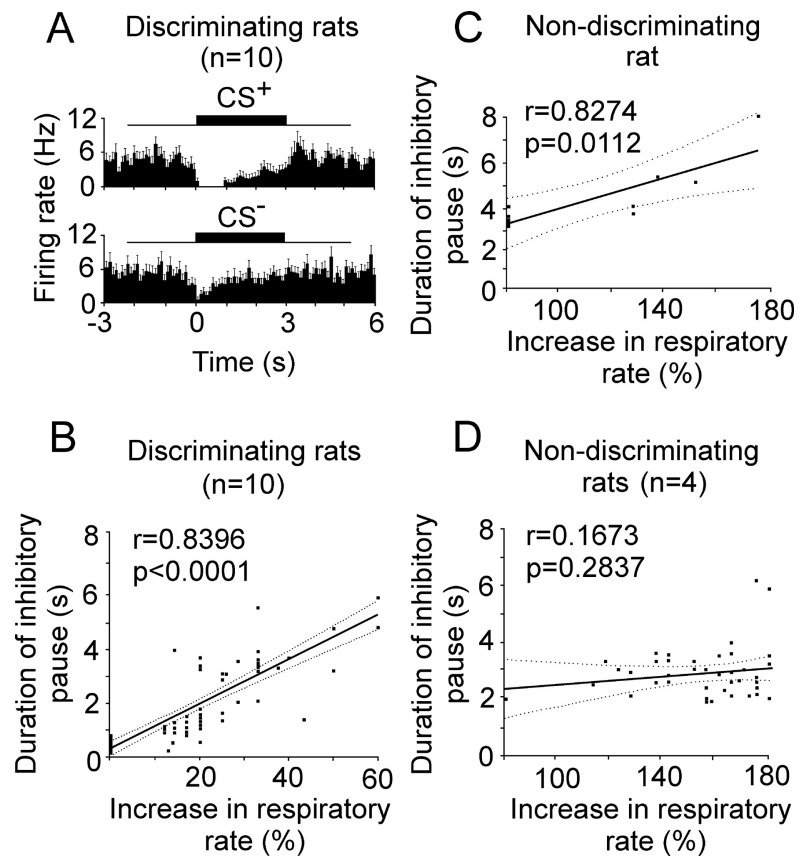

Figure 4. The duration of inhibition of VTA DA neurons depends on a level of conditioned fear. $A$, Population peristimulus time histograms $(100 \mathrm{~ms}$ bin) illustrating the responses of 10 $\mathrm{DA}$ neurons to the $\mathrm{CS}^{+}$and $\mathrm{CS}^{-}$during the initial six trials that were characterized by stable respiratory responses to the $\mathrm{CS}^{+}$in discriminating rats. $\boldsymbol{B}$, The correlation between the duration of inhibitory pauses in the activity of DA neurons $(n=10)$ and the increase in respiratory rate during presentations of the $\mathrm{CS}^{+}$(7-13 trials) evoking partial extinction of respiratory responses in discriminating rats. The correlation is expressed by a linear regression: $y=0.3398$ $+0.0792 x ; F=160.05, p<0.0001, \mathrm{df}=1,67$. C, The correlation between the duration of inhibitory pauses in the activity of a DA neuron and the increase in respiratory rate in one non-discriminating rat $(y=0.92070+0.03207 x ; F=13.02, p=0.0112, \mathrm{df}=1,8)$. $\boldsymbol{D}$, The lack of correlation between the duration of inhibitory pauses in the activity of $D A$ neurons $(n=$ 4) and the increase in respiratory rate in four non-discriminating rats ( $y=1.694+0.007 x$; $F=1.180, p=0.2837, \mathrm{df}=1,41)$. Each black square represents one trial without shock delivery.

spikes in these three neurons correlated with the acoustic startle responses evoked by the $\mathrm{CS}^{+}$onset.

To determine whether different levels of fear modulate inhibitory responses of VTA DA neurons, we analyzed their activity in 10 discriminating rats. We found that the $\mathrm{CS}^{+}$evoking a higher increase in respiratory rate than the $\mathrm{CS}^{-}$elicited significantly longer inhibition of DA neurons (Fig. $4 A$ ) and longer inhibitory pauses in their activity $(2.47 \pm 0.45 \mathrm{~s}$, minimum $=1.28 \mathrm{~s}$, maximum $=3.67 \mathrm{~s}$ vs $0.92 \pm 0.25 \mathrm{~s}$, minimum $=0.33 \mathrm{~s}$, maximum $=$ $2.09 \mathrm{~s}$; paired $t$ test, $t=3.87, p=0.0038, \mathrm{df}=9$ ). The DA cell inhibition to the $\mathrm{CS}^{+}$was followed by a significant (paired $t$ test, $t=7.791, p<0.0001, \mathrm{df}=9)$ increase in firing rate $(42 \pm 6 \%$, minimum $=15 \%$, maximum $=69 \%, n=10)$ compared with the baseline level. This rebound excitation of DA neurons lasted $\sim 1 \mathrm{~s}$ after the $\mathrm{CS}^{+}$offset (Fig. 4A) and was not observed after the $\mathrm{CS}^{-}$ termination (paired $t$ test, $t=1.748, p=0.1144, \mathrm{df}=9$ ).

To study the relationship between the within-session fear extinction and inhibition of DA neurons, we estimated the correlation between respiratory rates and the duration of inhibitory pauses in the activity of DA neurons during consecutive presentations of the $\mathrm{CS}^{+}$evoking a gradual decrease in respiratory responses (supplemental Fig. S1 A, trials 7-13, available at www. jneurosci.org as supplemental material). We found that the correlation between the duration of inhibitory pauses in DA neural activity and the increase in respiratory rate elicited by the delivery of the $\mathrm{CS}^{+}$was well described by a linear function with a positive slope (Fig. $4 B$ ). In the group of rats $(n=5)$ that did not discriminate between the CSs and expressed continuous freezing behavior, one rat showed a positive correlation between the duration of inhibitory pauses of a DA neuron and the increase in respiratory rate during $\mathrm{CS}^{+}$presentations (Fig. $4 \mathrm{C}$ ). In the other four non-discriminating rats, this correlation was insignificant because of the lack of extinction of respiratory responses to the $\mathrm{CS}^{+}$delivery and relatively stable inhibitory pauses in responses of DA neurons (Fig. 4D). Importantly, the average duration of inhibitory pauses in responses of DA neurons to the $\mathrm{CS}^{+}$during the initial six trials was significantly shorter in discriminating rats $(n=10)$ than in rats $(n=5)$ that did not distinguish between the CSs $(2.47 \pm 0.45 \mathrm{~s}$, minimum $=1.28 \mathrm{~s}$, maximum $=3.67 \mathrm{~s}$ vs $3.2 \pm 0.32 \mathrm{~s}$, minimum $=2.8 \mathrm{~s}$, maximum $=4.72 \mathrm{~s}$; MannWhitney test, $p=0.037$ ).

To further validate a correlation between inhibition of DA neurons and conditioned fear, we recorded the activity of VTA DA neurons in two rats after fear extinction and six naive rats habituated to light restraint. We found that presentations of the $\mathrm{CS}^{+}$after extinction of respiratory responses did not elicit inhibition of DA neurons (Fig. 5). Similarly, the delivery of auditory stimuli unpaired with electrical shock did not change either the breathing rhythm or firing rate of DA neurons recorded in naive rats after habituation to light restraint (Fig. 6).

\section{Discussion}

We found that the CSs associated with electrical shock evoked three types of biphasic responses in neurochemically identified VTA DA neurons. The presence of inhibitory pauses in these responses suggests that the transient suppression of DA neural activity is specifically related to acute fear evoked by the delivery of the $\mathrm{CS}^{+}$. Indeed, we found that the $\mathrm{CS}^{+}$elicited significantly longer inhibition of DA neurons and higher acceleration of breathing than the $\mathrm{CS}^{-}$. Furthermore, the duration of inhibitory pauses of DA neurons was in direct proportion to the increase in respiratory rate. It has been demonstrated that acceleration of breathing as well as the elevation in blood pressure and heart rate reflect an increased level of fear in rats during anticipation of electrical shock (Frysztak and Neafsey, 1991, 1994). Thus, our results suggest that VTA DA neurons are involved in distinguishing between the CSs having different aversive values, and that the duration of inhibition of DA neurons encodes a level of fear evoked by aversive stimuli. In four of the five freezing rats, which did not discriminate between the $\mathrm{CS}^{+}$and $\mathrm{CS}^{-}$, the linear correlation between the duration of inhibitory pauses of DA neurons and the increase in respiratory rate was not revealed because of the lack of within-session fear extinction. These rats expressed continuous freezing behavior and strongly accelerated breathing in response to each $\mathrm{CS}^{+}$delivery. We suggest that the relatively stable duration of inhibitory pauses, which we detected in the responses of DA neurons to consequent CSs in rats expressing continuous freezing behavior, encodes intense persisting fear in this group of animals. It was previously shown that rats highly sensitive to stress are characterized by prolonged freezing behavior in response to a tone predicting electrical shock, and that this freezing behavior is resistant to extinction (Shumake et al., 2005). In our study, fear conditioning was performed in lightly restrained rats; therefore, we cannot exclude the possibility that additional stress related to partial immobilization might modulate behavioral, respiratory, and DA neural responses to the CSs. Indeed, a body of evidence demonstrates that the preexposure of animals to stressful 
A

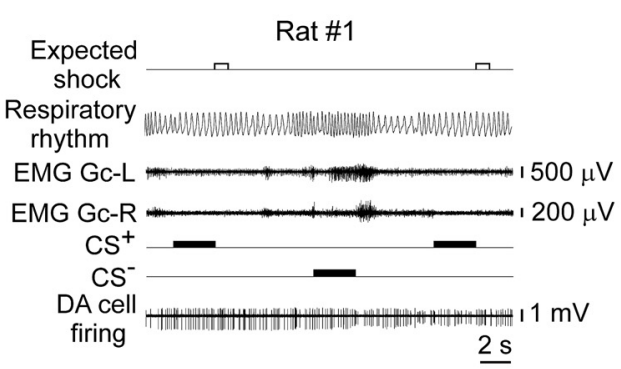

B

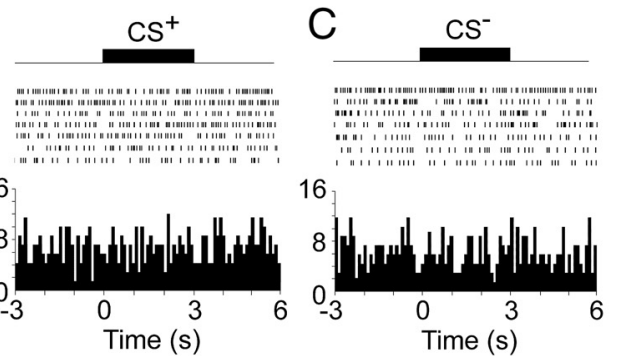

D

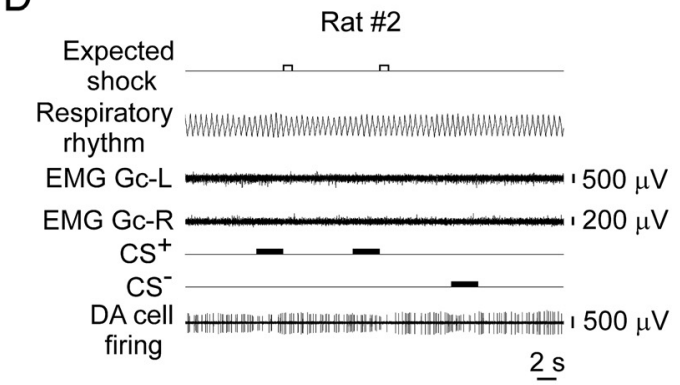

$\mathrm{E}$
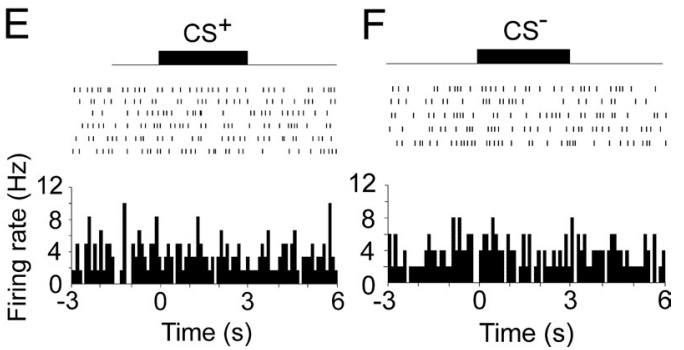

Figure 5. After fear extinction, neither the $\mathrm{CS}^{+}$nor $C S^{-}$presentations altered the discharge activity of VTA DA neurons or the respiratory rate in rats. $A, D$, Lack of respiratory acceleration and inhibitory responses of $D A$ neurons to the $C S^{+}$or $C S^{-}$presentations. $\boldsymbol{B}, \boldsymbol{C}, \boldsymbol{E}, \boldsymbol{F}$, Spike raster plots and peristimulus time histograms illustrating the lack of responses of these neurons to the $\mathrm{CS}^{+}$or $C S^{-}$during consequent trials. EMG GC-L, Electromyograms of the left gastrocnemius muscles; EMG GC-R, electromyograms of the right gastrocnemius muscles.

events, including restraint, substantially facilitates subsequent classic fear conditioning (for review, see Shors, 2004).

In our experiments, the inhibition to the $\mathrm{CS}^{+}$observed in the majority of VTA DA neurons was followed by a transient increase in their firing rate. Short-term excitation of neurochemically confirmed DA neurons was also reported in anesthetized rats after termination of nociceptive stimulation inhibiting their discharge activity (Brischoux et al., 2009). The transient increase in DA cell firing that follows inhibition may explain the elevation in DA release detected by microdialysis in the medial prefrontal cortex and limbic structures in response to aversive unconditioned and conditioned stimuli (Abercrombie et al., 1989; Puglisi-Allegra et al., 1991; Kalivas and Duffy, 1995). Since the microdialysis technique lacks high temporal resolution, brief reductions in DA release corresponding to inhibitory DA cell
A

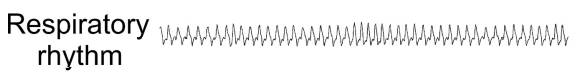

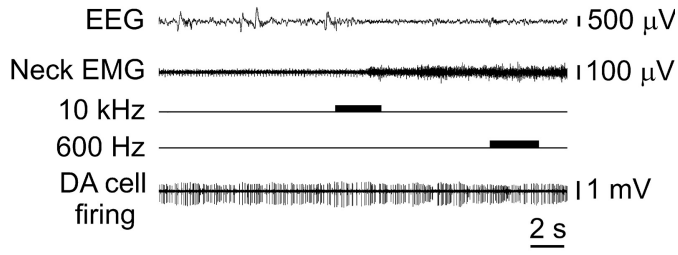

B

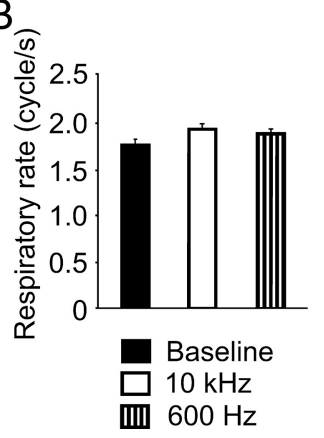

C

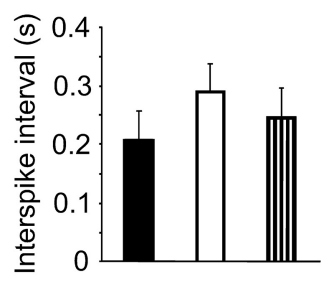

$\square 10 \mathrm{kHz}$

Figure 6. Presentations of auditory stimuli unpaired with electrical shock do not elicit the acceleration of a respiratory rhythm or inhibitory responses in DA neurons recorded in naive rats habituated to light restraint. $\boldsymbol{A}$, The first presentation of the $10 \mathrm{kHz}$ tone $(70 \mathrm{~dB})$ during drowsiness elicited EEG desynchronization and weak acceleration of the respiratory rhythm without virtual inhibition of a DA neuron. The subsequent presentation of the $600 \mathrm{~Hz}$ tone $(70 \mathrm{~dB})$ did not change either the respiratory rhythm or the baseline DA cell firing. $\boldsymbol{B}$, The average respiratory rates recorded in six control rats before (baseline) and during the presentation of $10 \mathrm{kHz}$ or 600 Hz tones. One-way ANOVA, $F=0.6472, p=0.5375, \mathrm{df}=2,15$. C, The average interspike intervals calculated for 3 s before (baseline) and after the onset of $10 \mathrm{kHz}$ or $600 \mathrm{~Hz}$ tones for six DA neurons recorded in six control rats. One-way ANOVA, $F=0.5281, p=0.6003, \mathrm{df}=2,15$.

responses to aversive stimuli have not been detected in these experiments. A recent study using fast scan cyclic voltammetry have shown that aversive taste stimulation elicited a sharp decrease in the DA level in the nucleus accumbens for 4-5 s (Roitman et al., 2008).

Different salient events, such as novel, unexpected, and highintensity sensory stimuli, evoke fast phasic excitatory responses in DA neurons (Horvitz et al., 1997; Horvitz, 2000; Schultz, 2007; Redgrave et al., 2008). We found that a small group of VTA DA neurons $(n=3)$ exhibited biphasic excitatory/inhibitory responses to aversive CSs. Since spikes elicited in these DA neurons had very short latencies ( $18-45 \mathrm{~ms})$, excitatory responses might reflect sensory or motivational salience of the CSs, whereas inhibitory pauses following short-latency excitation may encode the negative emotional value of the CSs (for review, see BrombergMartin et al., 2010). Two of the three DA neurons were located in the parabrachial pigmented nucleus, and the third neuron was situated in the paranigral nucleus of the VTA. Brischoux et al. (2009) showed the locations of four of five DA neurons excited by stimulation of nociceptive inputs in anesthetized rats in the paranigral pigmented and parainterfascicular nuclei of the VTA and one neuron in the parabrachial pigmented nucleus. However, the sample sizes in both studies are insufficient to make any conclusion about the predominant locations of these groups of DA neurons in the VTA.

It is well documented that DA neurons are involved in forming positive and negative prediction error signaling in rewardrelated paradigms (Mirenowicz and Schultz, 1996; Bayer et al., 2007; Schultz, 2007; Matsumoto and Hikosaka, 2009a). These neurons transiently increase their activity if the reward exceeds 
expectations and decrease or stop firing if the reward is omitted or has less value than expected. Bayer et al. (2007) reported that pauses in the activity of putative DA neurons became longer for more negative reward prediction errors and that this correlation had a largely linear character. Together with our results, these data suggest the involvement of similar neural circuits in monotonic encoding of both the magnitude of negative reward prediction error and the negative emotional value of the CSs. Indeed, recent studies show that inhibition of DA neurons during reward omission and presentation of aversive stimuli may be mediated by neurons of the lateral habenula (Matsumoto and Hikosaka, 2009b) and rostromedial tegmental nucleus (Jhou et al., 2009). Neurons in these structures showed excitatory responses to both reward omission and aversive stimulation and decreased their firing in response to rewarding stimuli.

It was hypothesized that the increase in DA signaling is associated with the motivated go process or action (Romo and Schultz, 1990; Schultz and Romo, 1990; Ljungberg et al., 1992). If so, the transient decrease in DA concentration evoked by inhibition of VTA DA neurons in response to aversive stimuli might facilitate behavioral arrest during processing of sensory signals associated with fear. In contrast, long-lasting inhibition of DA cell activity evoked by events with excessive negative values might reflect transient disintegration of behavior and the loss of adaptive responding to environmental stimuli.

Inhibition of VTA DA neurons by negative CSs also suggests the participation of these neurons in forming an aversive teaching signal mediating avoidance learning (Johansen and Fields, 2004) via interaction with the lateral habenular (Matsumoto and Hikosaka, 2009b) and rostromedial tegmental nucleus (Jhou et al., 2009). A recent study (Brinschwitz et al., 2010) showed that lateral habenular glutamate neurons may convey aversive/negative information to rostromedial tegmental GABA neurons that inhibit VTA DA neurons (Jhou et al., 2009). Transient reduction in DA signaling, in turn, may affect different brain systems mediating aversive learning.

Thus, our results show that the duration of inhibition of VTA DA neurons encodes a level of fear evoked by the CSs in a monotonically increased manner in fear-conditioned rats. The ability of VTA DA neurons to encode stimuli with different negative emotional values suggests the participation of these neurons in the analysis of signals that predict the degree of aversiveness of events in the environment.

\section{References}

Abercrombie ED, Keefe KA, DiFrischia DS, Zigmond MJ (1989) Differential effect of stress on in vivo dopamine release in striatum, nucleus accumbens, and medial frontal cortex. J Neurochem 52:1655-1658.

Bayer HM, Lau B, Glimcher PW (2007) Statistics of midbrain dopamine neuron spike trains in the awake primate. J Neurophysiol 98:1428-1439.

Brinschwitz K, Dittgen A, Madai VI, Lommel R, Geisler S, Veh RW (2010) Glutamatergic axons from the lateral habenula mainly terminate on GABAergic neurons of the ventral midbrain. Neuroscience 168:463-476.

Brischoux F, Chakraborty S, Brierley DI, Ungless MA (2009) Phasic excitation of dopamine neurons in the ventral VTA by noxious stimuli. Proc Natl Acad Sci U S A 106:4894-4899.

Bromberg-Martin ES, Matsumoto M, Hikosaka O (2010) Dopamine in motivational control: rewarding, aversive, and alerting. Neuron 68:815-834.

Frysztak RJ, Neafsey EJ (1991) The effect of medial frontal cortex lesions on respiration, "freezing" and ultrasonic vocalizations during conditioned emotional responses in rats. Cereb Cortex 1:418-425.

Frysztak RJ, Neafsey EJ (1994) The effect of medial frontal cortex lesions on cardiovascular conditioned emotional responses in the rat. Brain Res 643:181-193.

Gallego J, Nsegbe E, Durand E (2001) Learning in respiratory control. Behav Modif 25:495-512.

Gasbarri A, Verney C, Innocenzi R, Campana E, Pacitti C (1994) Mesolimbic dopaminergic neurons innervating the hippocampal formation in the rat: a combined retrograde tracing and immunohistochemical study. Brain Res 668:71-79.

Guarraci FA, Kapp BS (1999) An electrophysiological characterization of ventral tegmental area dopaminergic neurons during differential Pavlovian fear conditioning in the awake rabbit. Behav Brain Res 99:169-179.

Horvitz JC (2000) Mesolimbocortical and nigrostriatal dopamine responses to salient non-reward events. Neuroscience 96:651-656.

Horvitz JC, Stewart T, Jacobs BL (1997) Burst activity of ventral tegmental dopamine neurons is elicited by sensory stimuli in awake cats. Brain Res 759:251-258.

Jhou TC, Fields HL, Baxter MG, Saper CB, Holland PC (2009) The rostromedial tegmental nucleus (RMTg), a GABAergic afferent to midbrain dopamine neurons, encodes aversive stimuli and inhibits motor responses. Neuron 61:786-800.

Johansen JP, Fields HL (2004) Glutamatergic activation of neurons in the anterior cingulate cortex is necessary and sufficient to produce an aversive teaching signal. Nat Neurosci 7:398-403.

Kalivas PW, Duffy P (1995) Selective activation of dopamine transmission in the shell of the nucleus accumbens by stress. Brain Res 675:325-328.

Ley R (1999) The modification of breathing behavior: Pavlovian and operant control in emotion and cognition. Behav Modif 23:441-479.

Ljungberg T, Apicella P, Schultz W (1992) Responses of monkey dopamine neurons during learning of behavioral reactions. J Neurophysiol 67:145-163.

Luo AH, Georges FE, Aston-Jones GS (2008) Novel neurons in ventral tegmentral area fire selectively during the active phase of the diurnal cycle. Eur J Neurosci 27:408-422.

Matsumoto M, Hikosaka O (2009a) Two types of dopamine neurons distinctly convey positive and negative motivational signals. Nature 459:837-841.

Matsumoto M, Hikosaka O (2009b) Representation of negative motivational value in the primate lateral habenula. Nat Neurosci 12:77-84.

Mileykovskiy BY, Kiyashchenko LI, Siegel JM (2002) Cessation of activity in red nucleus neurons during stimulation of the medial medulla in decerebrate rats. J Physiol 545:997-1006.

Mirenowicz J, Schultz W (1996) Preferential activation of midbrain dopamine neurons by appetitive rather than aversive stimuli. Nature 379:449-451.

Paxinos G, Watson C (1997) The rat brain in stereotaxic coordinates. New York: Academic.

Puglisi-Allegra S, Imperato A, Angelucci L, Cabib S (1991) Acute stress induces time-dependent responses in dopamine mesolimbic system. Brain Res 554:217-222.

Redgrave P, Gurney K, Reynolds J (2008) What is reinforced by phasic dopamine signals? Brain Res Rev 58:322-339.

Roitman MF, Wheeler RA, Wightman RM, Carelli RM (2008) Real-time chemical responses in the nucleus accumbens differentiate rewarding and aversive stimuli. Nat Neurosci 11:1376-1377.

Romo R, Schultz W (1990) Dopamine neurons of the monkey midbrain: contingencies of responses to active touch during self-initiated arm movements. J Neurophysiol 63:592-606.

Schultz W (2007) Behavioral dopamine signals. Trends Neurosci 30:203-210.

Schultz W, Romo R (1990) Dopamine neurons of the monkey midbrain: contingencies of responses to stimuli eliciting immediate behavioral reactions. J Neurophysiol 63:607-624.

Shors TJ (2004) Learning during stressful times. Learn Mem 11:137-144.

Shumake J, Barrett D, Gonzalez-Lima F (2005) Behavioral characteristics of rats predisposed to learned helplessness: reduced reward sensitivity, increased novelty seeking, and persistent fear memories. Behav Brain Res 164:222-230.

Swanson LW (1982) The projections of the ventral tegmental area and adjacent regions: a combined fluorescent retrograde tracer and immunofluorescence study in the rat. Brain Res Bull 9:321-353.

Ungless MA, Magill PJ, Bolam JP (2004) Uniform inhibition of dopamine neurons in the ventral tegmental area by aversive stimuli. Science 303: $2040-2042$. 